\title{
At the Crossroads of Slavonic Compilations
}

\author{
William R. Veder
}

Indepedent scholar, Utrecht, The Netherlands

veder@sbcglobal.net

\begin{abstract}
Close examination of five compilations from texts previously translated into Slavonic (two of which are edited) and their interrelation leads to the recognition that they form part of a corpus with at least another eleven compilations (four of which are edited). This corpus, in size vastly surpassing that of original compositions in Slavonic, was written in Glagolitic script, which implies that it cannot have been created later than the beginning of the twelfth century.
\end{abstract}

\section{Keywords}

(Old Church) Slavonic - Glagolitic - Compilation from previous translations Compilation technique

Literary scholarship usually treats compilations as the poor relations of 'real' literature for being limited to recycling texts created previously and muddling their associations, composition and meaning. Not so Norman Ingham: he drew my attention to the striking fact that the Scaliger Patericon of the tenth century was compiled using a technique of chaotisation, which came into its own only with computer-generated poetry at the end of the twentieth century. ${ }^{1}$ I owe it to his memory to show that compilations have much insight to offer to the attentive reader and ought to be recognised as a valuable source for the history of language, literature and culture, some of them even as art in their own right.

1 See W.R. Veder, 'Literature as a Kaleidoscope: the Structure of Chetii Sborniki,' in U.R. Feder, Hiliada godini kato edin den: zhivot"t na tekstovete $v$ pravoslavnoto slavianstvo (Sofia: Marin Drinov, 2005), 108. 


\section{An Exhortation on Reading}

The third-oldest dated book in Rus', the Izbornik of $1076,{ }^{2}$ is unlike the Ostromir Gospel Lectionary of 1056-57 and the Izbornik of 1073 (copied from the Simeonov Izbornik ${ }^{3}$ ) not a translated Greek but a Slavonic compilation (copied from the Izbornik of John the Sinner ${ }^{4}$ ). It opens with a well-known and very wellwrought ${ }^{5}$ exhortation on reading. I print it below under the siglum ' $\mathrm{g}$ ' in normalised spelling and collate it with the Zlatoust (siglum ' $z$ '). ${ }^{6}$ To $g$ and $\mathrm{z}$ I add their identified sources: $\mathrm{i}$ - the Izmaragd, version b, chapters 2 and $104,{ }^{7} \mathrm{k}$ - the Kniazhii Izbornik ${ }^{8}$ and s - the Scete Patericon, version + , chapters $\mathrm{G}$ and $1 .^{9}$

\footnotetext{
$o$ (s G:1:1)

S Рече Блажеnzin Iwanznz ZалтооустZін.

і САОВО СВАТАКГГ ГРНГООННА ПАПЫ РНМЬСКАКГО.

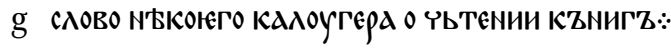

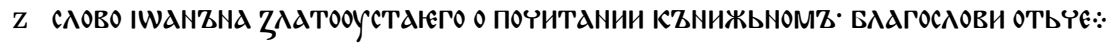
1 (i 2:1)

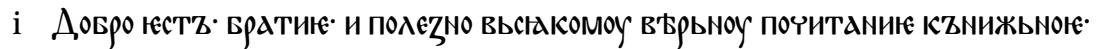

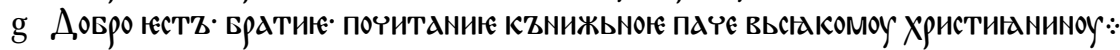

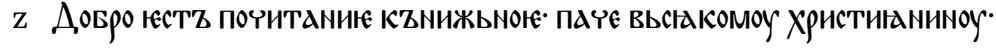
2 (i 2:2, Ps 118:2, 10)
}

2 New edition by M.S. Mushinskaia et al., Izbornik 1076 goda. Vtoroe izdanie, pererabotannoe $i$ dopolnennoe, T. 1-2, (Moskva: Rukopisnye pamiatniki Drevnei Rusi, 2009).

3 Ed. P. Dinekov et al., Simeonov sbornik (po Svetoslavoviia prepis ot 1073 g.), Vol. 1-3, (Sofia: Izd. Na Balgarskata Akad. na Naukite, 1991-2015).

4 Ed. U.R. Feder, Kniazhii Izbornik za v"zpitanie na kanartikina, Vol. 2, (Veliko T"rnovo: Sv. Sv. Kiril i Metodi, 2008); English translation in W.R. Veder, A.A. Turilov, The Edificatory Prose of Kievan Rus' (Cambridge, Mass.: Harvard Ukrainian Research Institute, 1994) (= Harvard Library of Early Ukrainian Literature, English Translations, Vol. vI).

5 See W.R. Veder, 'Three Old Slavic Discourses on Reading,' in Feder, Hiliada..., 130-138; for another well-wrought compilation, see Ya. Miltenov, 'Novi danni za Pouchenieto za spasenieto na dushata, pripisano na Chernorizec Pet"r,' Starob"lgarska literatura $5^{1}$ (2015): 157-187.

6 In a reconstruction of the antigraph of two 16th c. copies in the Trinity-St Sergius Laura, viz. codd. Moskva RG B F.173.I nr. 146, f. $334 \mathrm{v}-338$ and F.304.I nr 142, f.221v-224v.

7 In a reconstruction of the antigraph of three $15^{-16 t h ~ c . ~ c o p i e s ~ i n ~ t h e ~ T r i n i t y-S t ~ S e r g i u s ~ L a u r a, ~}$ viz. codd. Moskva RG B F.173.I nr. 46, f.8-v, F.304.I nr. 202, f.2v-3v, and nr. 203, f.5-v.

8 Ed. Feder, Kniazhii..., Vol. 2.

9 Ed. W.R. Veder, Skitskij Paterik, Amsterdam 2012 (= Pegasus Oost-Europese Studies 14); for the compilation, see U.R. Feder, 'Kniazhij Izbornik i Izbornik na greshniia Joan sred rannite glagolicheski kompilacii,' in: A. Angusheva et al. (eds.). Vis et sapientia: Studia in honorem Anisavae Miltenova, (Sofia: Boian Penev, 2016), 395-419. 


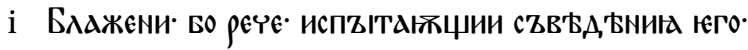

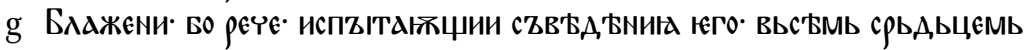

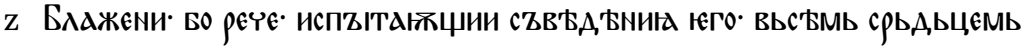
i

g вZZнцжтZ юго:

Z вZZZІщжТZ юего. 3 (Ps 118:11)

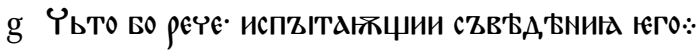

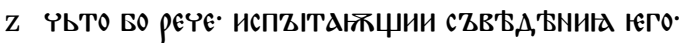
4 (s G:1:3-4)

S КЕГАА ЖЕ ЧЬТЕШИ... NЕ ТZЧИ СА АНСТЫІ ТZКZМО ОБРАЩАТИ·

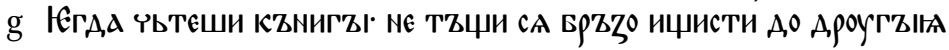

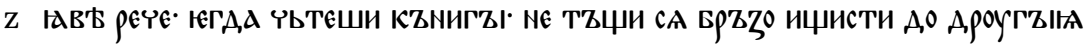

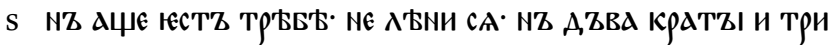

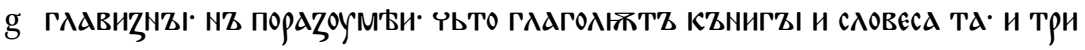

Z ГААВИZ

s краты прочитан грами·

g КРАТЫ ОБРАЧАН СА О КА,ИНОИ ГААВНZНЪ:

Z КОАТЫ ОБРАЩАИ СА О ЮА,ИНОН ГААВНZN'В. 5 (i 2:8, Ps 118:11)

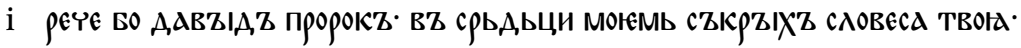

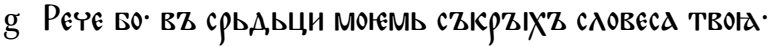

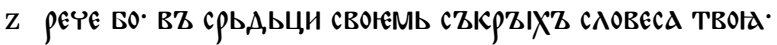

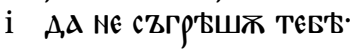

g A,A NE сZГр'Бшж ТеБ':

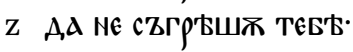
6 (Ps 118:11)

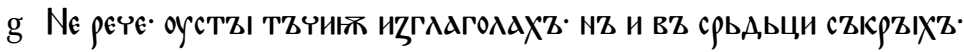
A, NE СZГр'ВШж TEБЪ:

z 10 7

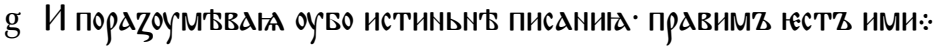

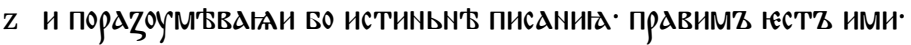
8 (i 2:9, Jas 3:3)

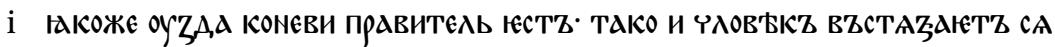

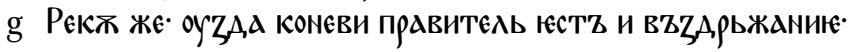

10 The identical ending of verses 5 and 6 probably caused the accidental omission in $\mathrm{z}$. 


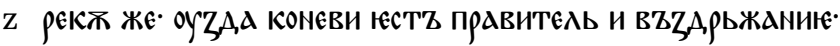

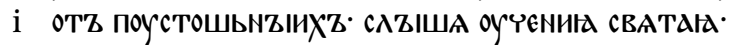

g ПрАВЬА,ЬАИКОУ ЖЕ КZАИГZ1:

Z ПрАВЬА,ЬАнКОУ Же КZNИГZ'

9 (Jas 3:4)

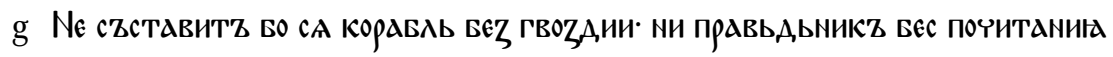

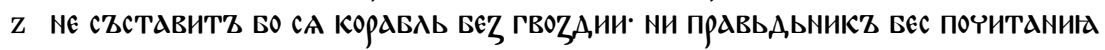

g КZNНЖЬНАКГО:

Z КZNИЖЬНАКГО:

10 (i 2:10)

i И ґаКОЖе П

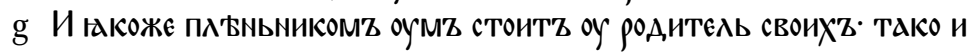

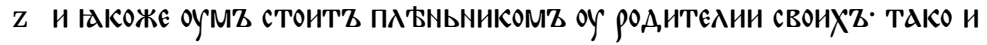

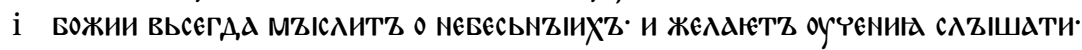

g ПрАВЬА,ЬNИКСУ О ПОЧИТАКИИ КZNНЖЬN'ВМЬ:

Z ПРАВЬА,ЬNИКОМZ О ПОЧИТАNИИ КZNНЖЬМОМЬ•

11 (Ps 118:47)

g 11

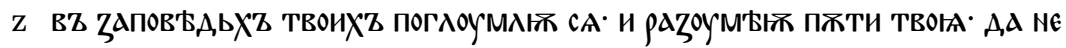

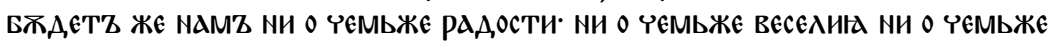
ГАОУМИТИ СА• NZ TZКZМО О ПОЧИТАКИИ КZNНЖЬМОМЬ•

12

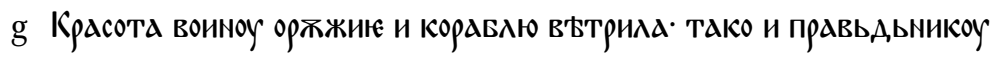

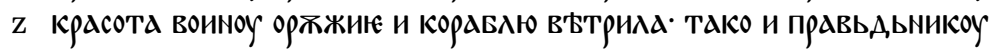

g ПОчИТАNИЕ КZNИЖЬNOК:

Z О ПОЧИТАКИИ КZNИЖЬNОМЬ

13 (s G:2:4, Ps 118:18)

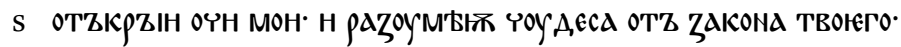

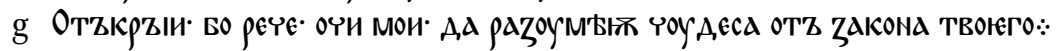

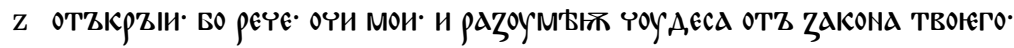
14 (Ps 118:19)

g ОЧИ БО ГААГОАЕТZ РАZОУМZ И СZMZICАZ СРЬАЬЧЬNZIН:

Z ОЧН БО ГААГОАЕТZ РАZОУМZ И СZMZIСАZ СРЬАЬЧЬNZIН.

15 (s G:2:3, Ps 118:19)

S NE СZКРZIИ ОТZ МЕNЕ ZАПОВБА,ИН ТВОНХZ.

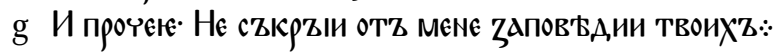

Z И ПРОЧЕКЕ NE СZКРZІИ ОТZ МЕNЕ ZАПОВ'АА, ИИ ТВОНХZ.

11 The identical ending of verses 11 and 12 probably caused the accidental omission in $i$. 
16

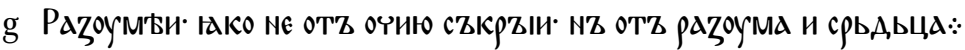

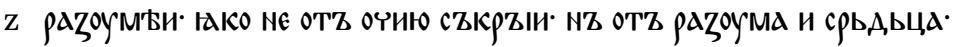
$17 a($ i $2: 11)$

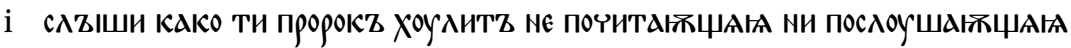
OY'SENHIA.

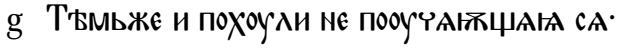

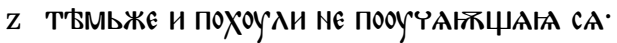
$i(\mathrm{i} \text { 104:1 })^{12}$

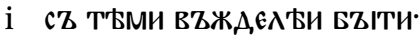

Z ТБХZ ЖЕ СА ТZІ ПРНЧАСТИТИ ВZЖА,АСНii (s $1: 3)$

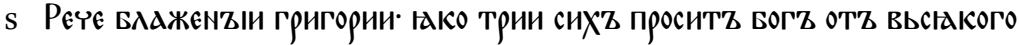

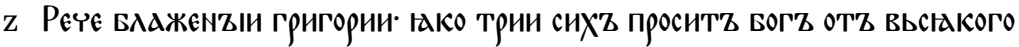

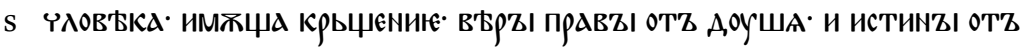

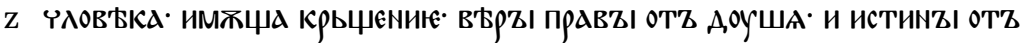

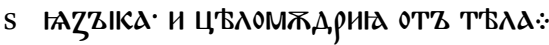

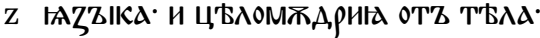
iii (i 104:2-4 = k 9:43-45, 1 Cor 2:9)

i ВЬСІа БО. W

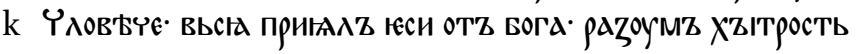

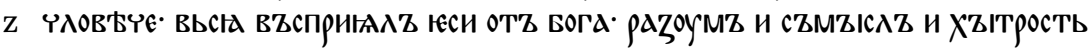

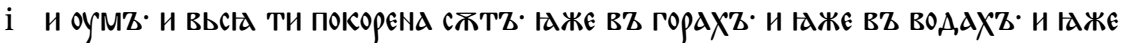

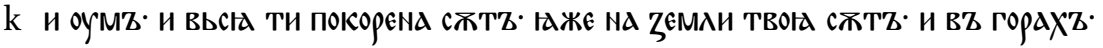

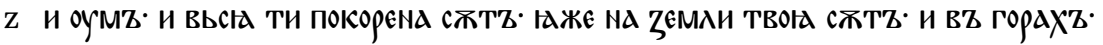

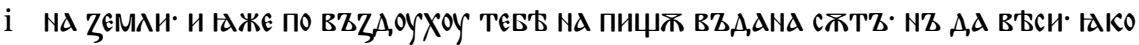

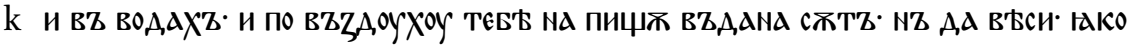

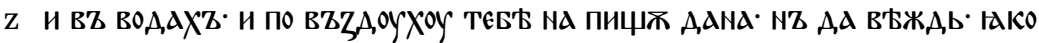

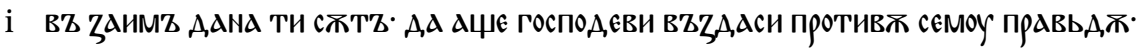

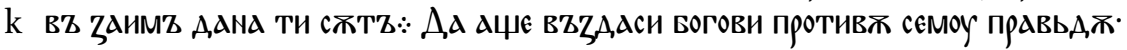

Z BZ ZАНMZ ААНА ТИ СЖTZ: АА АЩЕ ВZА,АСИ БОГОВИ ПРОТИВЖ СЕМОУ ПРАВЬА,

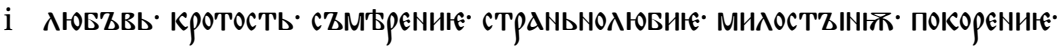

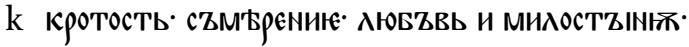

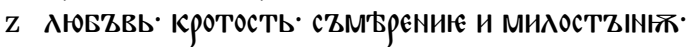

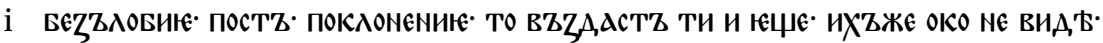

12 This verse - the last of Izmaragd b 104:1 (see interpolation $v$ below) - requires, of course, a positive antecedent. Its transfer to this place without negation is an error of the compiler of $\mathrm{i}$. 
k ТО ВZZААСТТZ ТЕББ ПАКZ ПРОТИВЖ ТОМОУ· ИХZЖЕ ОКО NЕ ВНА,'

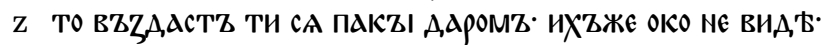

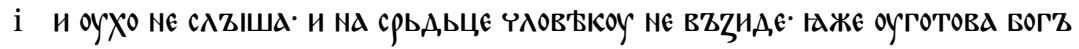

k

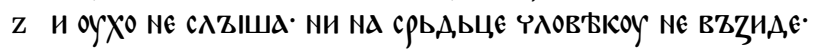

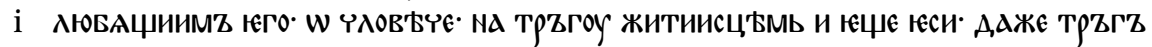

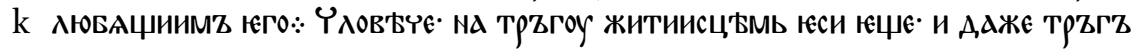

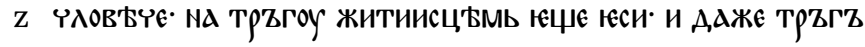

I СA NE РАZНА,ТTZ. КОУПИ СИ МНАОСТZINEIT ПОМНАОВАNИЕ ОТZ БОГА.

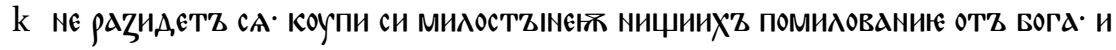

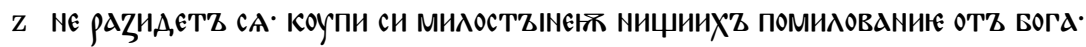

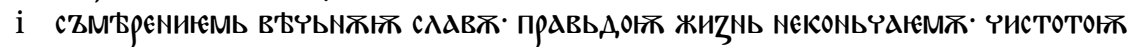

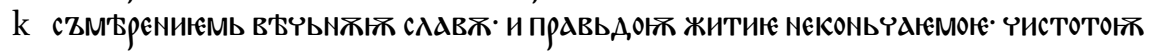

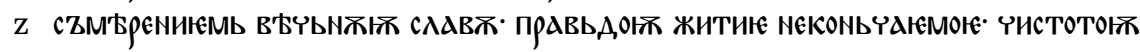

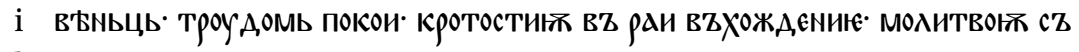

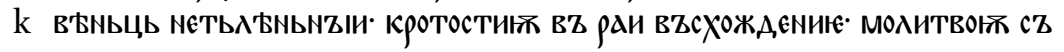

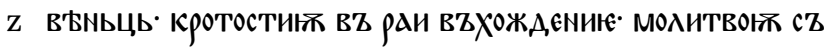

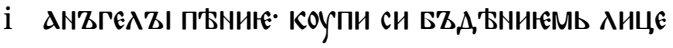

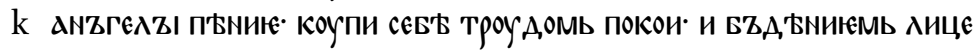

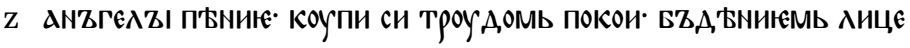

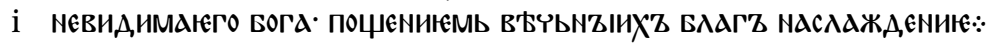

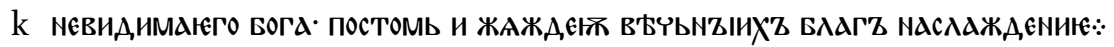

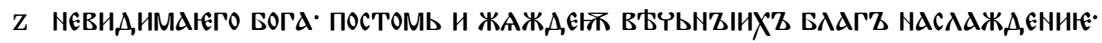
iv

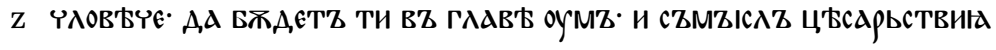

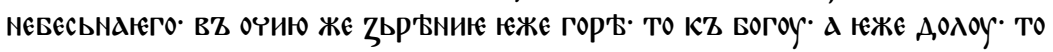

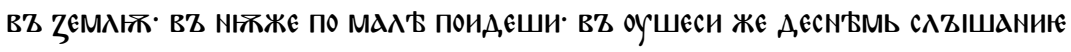

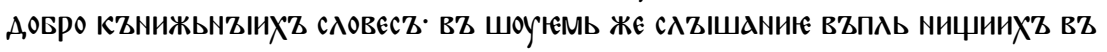

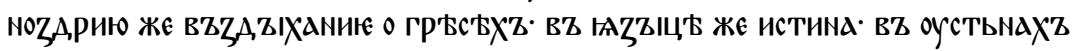

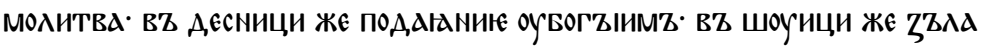

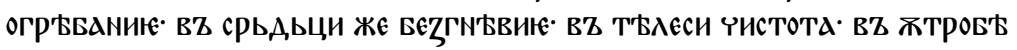
ААZКОТА· ВZ КО人'БNOY БОГОУ ПАА,ЕНИЕ И КZ БОГОУ ШЬСТВИК• МОАИТВЬNZIИ

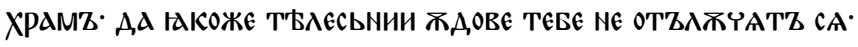
$17 b$ (i 2:12, Ps 118:21)

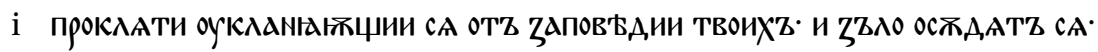

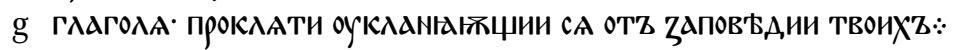

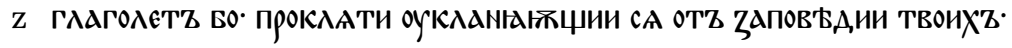
18 (i 2:13-14, Ps 118:103, 72)

I И САMZ СА ПОХВААИ ГААГОАА· КОЛЬ САААZКА ГРZТАNИ МОКЕМОУ САОВЕСА

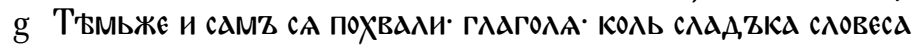


Z ТБМЬЖЕ И САMZ СА ПОХВАЛН ГААГОАА' КОЛЬ САААZКА ГРЬТАНИ МОКЕМОУ СЛОВЕСА

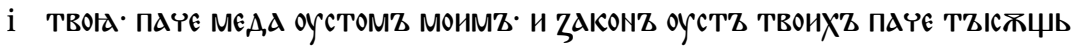

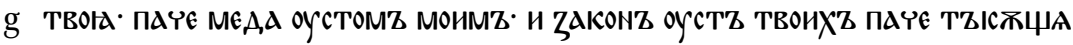

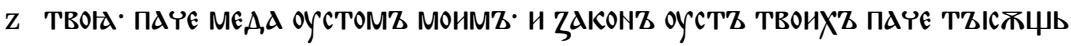

i ZАATA и сьревра.

g ZАATA и сьревра:

Z Z ZATA и сьревра. 19 (i 2:15, Ps 118:162)

В И ВZСПАТЬ ГААГОЛА ВZZА,РАА,ОУЮ СА АZZ О САОВЕСЬХZ ТВОИХZ ГАКО ОБР'БТАНА

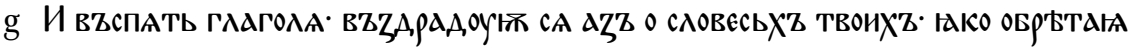

Z И ВZСПАТЬ ГААГОЛА. ВZZА,РАА,ОУЮ СА АZZ О САОВЕСЬХZ ТВОНХZ ЮАКО ОБРБТАҢА

i корнсть MZNOГж.

g корнсть MZNогж:

z корнсть мzногт. 20 (i 2:16, Ps 1:2)

i корнсть нарете словеса БОжина.

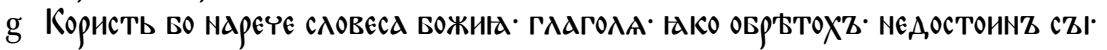

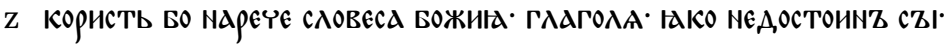

g ТАКZ ААРZ· КЖЕ МИ СА ПООУЧАТИ САОВЕСЬMZ ТВОНМZ А,ЬНЬ И КОЩЬ:

Z ТАКZ ААРZ ОБР'БТОХZ КЕЖЕ МИ СА ПООУЧАТИ САОВЕСЬМZ ТВОНМZ АЬНЬ И КОЩЬ 21 (s G:1:4)

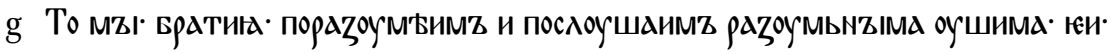

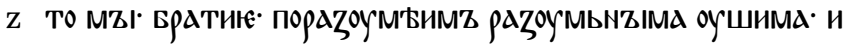

S AА раZоум'Веши сихж ихZ.

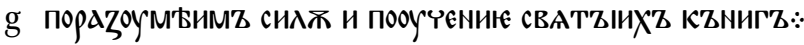

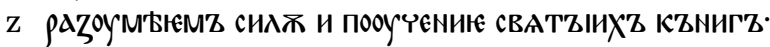
22 (i 2:19)

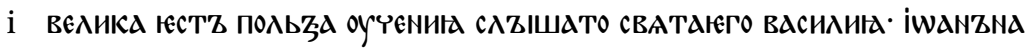

g ПослоуШаИ ТZІ ЖИТИН СВАТАКГО ВАСНАИН И СВАТАКГО IWANZNA

Z ПОСАОУШАН ТZІ ЖИТИҢ СВАТАКГО ВАСНАНІа И СВАТАКГО IWANZNA

і ZААтооустаюего є

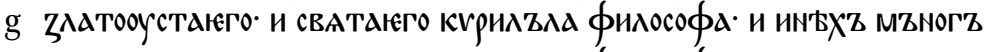

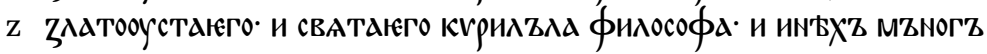

i CВATZIHXZ.

g СВАТЫИХZ:

Z CBATZIHXZ.

23

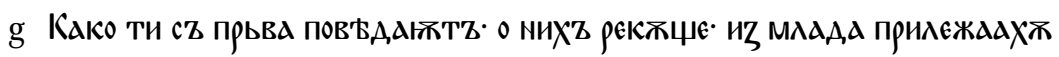

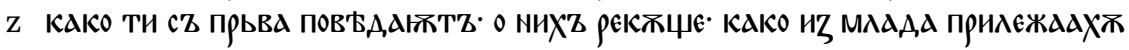

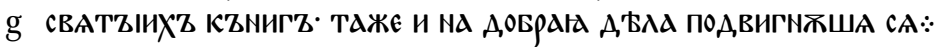


Z СВАТЫИХZ КZNИГАХZ· ТАЖАЕ И НА А,ОБОАҢ АТАА ПОАВИГКЖША СА• 24 (i 2:18)

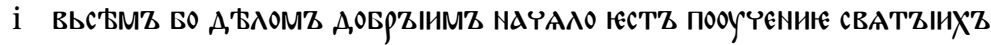

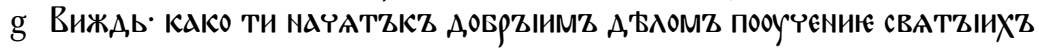

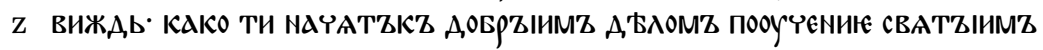

і КZNнГZ.

g КZNнгЪ:

Z КZNИГАMZ.

25

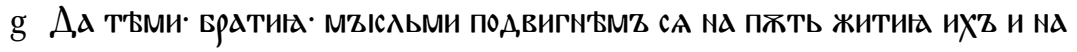

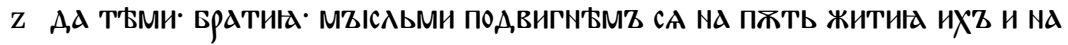

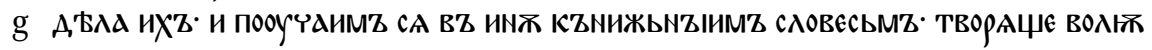

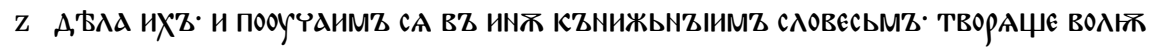

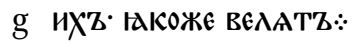

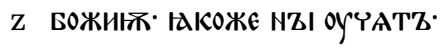
26 (i $2: 20)$

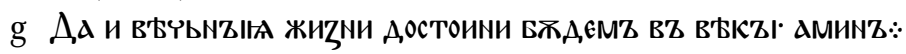

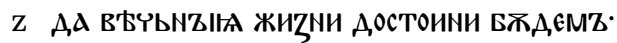

$v($ i 104:1)

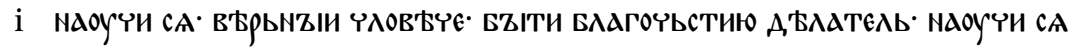

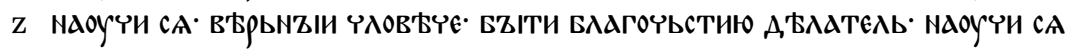

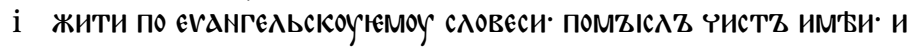

Z ЖИТИ ПО ЕVАКГе

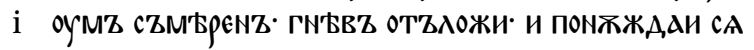

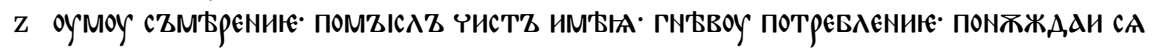

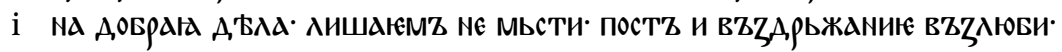

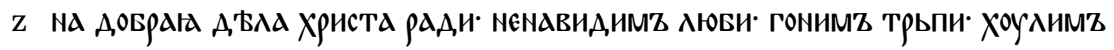

i NEMZNOГO СZПАNИК'

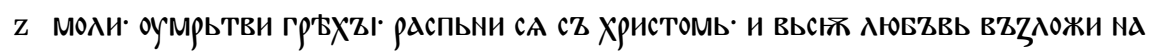

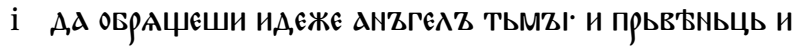

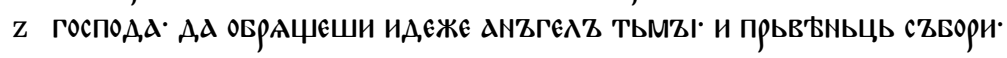

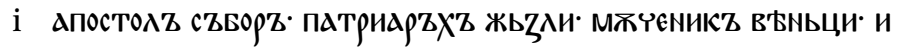

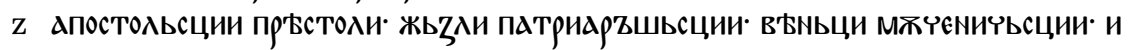

і поХВААА ПрАВЬА,ЬNZIИХZ.

z ПоХВААЫ ПРАВЬАЬМЗІМХZ.

$v i$

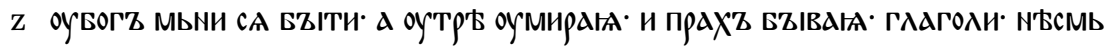

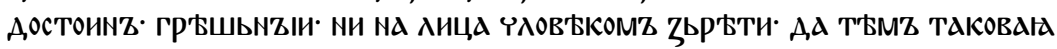

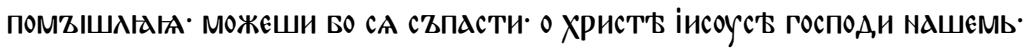
КЕМОУЖЕ СААВА И ЧЬСТЬ И АРЬЖАВА: 
We can see three successive stages of compilation: first, the sources, viz. i, $\mathrm{k}$ (Slavonic compilations) and s (a Greek compilation), second, g itself, and third, its recipient $\mathrm{z}$. But there is more than meets the eye, and a brief review of the closely related compilations, including one not yet mentioned, the Minejnyj Izbornik, ${ }^{13}$ is in order to get the full picture.

\section{A The Minejnyj Izbornik}

This compilation (ca. 38,00o words) contains a compilation from the Slavonic translations of the Capitula adhortatoria of Agapetus Diaconus, the Manuale of Epictetus in the version of Neilus of Ankyra, ${ }^{14}$ followed by 499 gnomes from works of an anonymous, a George, 'holy fathers', a Markianos, a Theodore, Basil the Great, Clement of Rome, Ephraem Syrus, Gregory of Nazianzus, Gregory of Nyssa, Hesychius of Jerusalem, Hypatius, Isaac of Niniveh, Isidore of Pelusium, the Apostle James, John Cassian, John Chrysostom, John Climacus, John Damascene, John of Karpathos, Maximus Confessor, Neilus of Ankyra, patriarchs Gennadius I and Photius, Palladius of Helenopolis, Zosimas and from the Vita of a St. Olympius, and concluded by the Prayer to the Virgin of Peter the Monk.

\section{B The Kniazhii Izbornik}

This compilation ${ }^{15}$ (ca. 30,00o words) draws upon the Minejnyj Izbornik (for the Capitula adhortatoria and the gnomes), adding gnomes gleaned from the Monostichis of Menander, the Simeonov Izbornik (a translated Greek compilation), the Izmaragd and the Slavonic translations of the Centuriae de caritate of Thalassius, the Quaestiones ad Antiochum of a Pseudo-Athanasius, the Quaestiones et responsiones of Anastasius of Sinai, the Sentences, variously attributed to Hesychius of Jerusalem or Neilus of Ankyra, the sermon De ascetica disciplina of Basil the Great, the Vitae of St. Niphon of Constantia, St. Theodora of Alexandria and St. Xenophon, the deuterocanonical Wisdom of Jesus, son of Sirach, and two texts perhaps translated, but possibly compiled in Slavonic, viz. a Centuria de fide and a Sermo patris ad filium suum. ${ }^{16}$

\footnotetext{
13 See D.M. Bulanin, 'Neizvestnyi istochnik Izbornika 1076 goda,' Trudy Otdela drevnerusskoi literatury 44 (1991): 161-178 and Mushinskaia, Izbornik..., Vol. 1, 52-54.

14 See D.M. Bulanin, Antichnye traditsii v drevnerusskoi literature XI-XVI vv, (München 1991) (= Slavistische Beiträge 278).

15 Ed. Feder, Kniazhii..., Vol. 2; for the compilation structure, see Vol. 1, 13-16.

16 Ed. W.R. Veder, 'A Certain Father's Edifying Words to His Son,' in Feder, Hiljada..., 139-144.
} 


\section{The Izbornik of John the Sinner}

The Izbornik of $1076^{17}$ is the only full copy of the Izbornik of John the Sinner (ca. 25,000 words). Its main source is the Kniazhii Izbornik (of its fifteen chapters, it copies ten without significant alteration and another three in abridged and reshuffled form), adding the exhortation (collated above), an excerpt from an unidentified source and gnomes from the Scete Patericon versions $\alpha$ and $\gamma$, the Scaliger Patericon, the Minejnyj Izbornik, ${ }^{18}$ homilies by John Chrysostom and Basil the Great and the Vita of St. Martin of Tours.

\section{The Izmaragd}

The Izmaragd is a compilation from a vast array of sources that exists in two loosely related versions, a (ca. 60,00o words) in ninety-two chapters and b (ca. 85,00o words) in 164 chapters. ${ }^{19}$ Both versions draw upon the same sources, translated from Greek (Barlaam and Joasaph, a set of gnomes from works of John Chysostom, the Epistle to Agathius of Neilus of Ankyra, the Pandect of Antiochus the Monk, the Paraenesis of Ephraem Syrus, the Quaestiones ad Antiochum of a Pseudo-Athanasius, the Scete Patericon, the Vita of St. Niphon of Constantia and others to be identified) and compiled or composed in Slavonic (the Discourse against the Bogumils of Cosmas the Priest, the Kniazhii or Minejnyj Izbornik, ${ }^{20}$ the Zlatostrui). To these, version a adds the Homilies of Cyril the Monk and the Simeonov Izbornik; version $\mathbf{b}$ adds the translations of the Asceticon of Isaiah of Gaza, the Dialogues of Gregory the Great, an epistle of Isidore of Pelusium, the Homilies on 1-2 Corinthians and Ephesians of John Chrysostom, the Homily on the Trinity by Didymus the Blind, homilies by Basil the Great and Pseudo-Eusebius, the Parable of the Snake attributed to

\footnotetext{
17 The structure of $\mathrm{C}$ is laid out in Feder, Kniazhii..., Vol. 1, 16-18.

18 The only independent excerpt from A is units 114-115 (see Mushinskaia, Izbornik..., Vol. 1, 218-219), all others are taken from B.

19 For version a, see B.M. Pudalov, 'Literaturnaia istoriia 1-oi ('Drevneishei') redaktsii Izmaragda,' Drevniaia Rus, Voprosy medievistiki 2000/2:76-95; for version b, B.M. Pudalov, 'K literaturnoi istorii sbornika Izmaragd: formirovanie 2-oi ('Osnovnoi') redaktsii. Trudy Otdela drevnerusskoi literatury 58 (2004): 330-342. In neither paper does he question the assumption of V.A. Iakovlev, K literaturnoi istorii drevnerusskikh sbornikov: Opyt issledovaniia Izmaragda,' (Odessa: np, 1893), that the compilations be assigned to 'Rus', 14th c.' A full-text collation of the versions in the Trinity-St Sergius Laura can be obtained by requesting the dossier 'Izmaragd' from <hilandar@osu.edu>.

For lack of an edition of A, I must refer to the edition of B.
} 
Christopher of Alexandria, the Quaestiones et responsiones of Anastasius of Sinai, the Pratum Spirituale of John Moschus (and others to be identified), as well as the Slavonic Izbornik of John the Sinner and homilies by Clement of Ohrid and Peter the Monk. In some chapters, version a appears to have drawn upon $\mathbf{b}^{21}$

Both versions differ from the compilations $\mathrm{A}-\mathrm{C}^{22}$ in that they are synthetic: they do not merely excerpt gnomes from their sources and concatenate them, but use them to create new texts. The attribution of texts so created is, of course, arbitrary, e.g. in version b, ch. 36 is compiled from B 9:45 + unidentified + Pandect of Antiochus ch. 60 and attributed to 'Basil the Great'; ch. $43=$ Quaestiones ad Antiochum $92+$ C 66-82 + B 3:30, 52 + unidentified $\rightarrow$ 'Athanasius'; ch. 56 = unidentified + B 9:177-178, 180-183, 179, $178 \rightarrow$ 'Chrysostom'; ch. 93 = unidentified + B 1:58, 67-78, 80-81 $\rightarrow$ 'Gennadius'; ch. $112=$ Quaestiones ad Antiochum $113 \rightarrow$ 'Holy Fathers'; ch. 155 = unidentified + Scete Patericon L:18-19 + unidentified $\rightarrow$ 'Chrysostom'. ${ }^{23}$

\section{E The Zlatoust}

The Zlatoust is a compilation (up to ca. 75,00o words) ordered following the ecclesiastical year, drawing upon both versions of the Izmaragd (eight chapters from version a and twenty-eight from version $\mathbf{b}$, eight entered twice on different dates), the Kniazhii Izbornik (two chapters), the Izbornik of John the Sinner (one chapter) as well as additional Slavonic sources. ${ }^{24}$ It is known in four versions, a Lenten (sixty-five chapters, including the weekdays), b Pentecostal (a + Sundays from Easter to Trinity Sunday, up to seventy-seven chapters), c Annual short (b, excluding the weekdays in Lent + Sundays after Trinity, 117 chapters) and $\mathbf{d}$ Annual long (b + Sundays after Trinity, up to 147 chapters). It shares with the Izmaragd the synthetic approach to compiling new texts.

21 See W.R. Veder, 'Gennadius Slavicus,' in: Srednovekovniiat chovek i negoviiat sviat, (Veliko T"rnovo: Faber, 2014), 273-285.

22 Rare exceptions are in $\mathrm{C}$ the exhortation and the additions to the excerpt from the Vita of St. Niphon of Constantia.

23 This explains the 'Gregory pope of Rome' in the title of the exhortation in i as well as the 'John Chrysostom' in z.

24 See O.V. Tvorogov, 'Opisaniie i klassifikatsiia spiskov sbornika Zlatoust,' Trudy Otdela drevnerusskoi literatury 39 (1985): 278-284 and O.V. Tvorogov and T.V. Chertoritskaia, 'Zlatoust,' in Slovar' knizhnikov i knizhnosti Drevnei Rusi, Vyp. 2, ch. 1, (Leningrad: Nauka, 1988): 397-401. The authors assign the compilation to 'Rus', late 15th - early 16th c.' A full-text collation of the versions in the Trinity-St Sergius Laura can be obtained by requesting the dossier 'Zlatoust' from <hilandar@osu.edu>. 
The vast array of sources, some of them available to two or more compilers for months on end, suggests that the compilations were made in a wellappointed library. The precision of excerption and recompilation suggests that the compilers had intimate knowledge of their sources, a knowledge that casual reading cannot impart. Slavonic requires writing to gain full access to a text: these compilers surely had written many of their sources, if only their copies, draft or fair.

\section{The Relation of Compilations $A-E$}

Compilations A-D share an indeterminacy of purpose, save, of course, for providing edifying reading matter: any explanation for their creation is as good as the next. A decade ago, I thought that the extremely restricted diffusion of $\mathrm{C}$ could be the result of its being kept out of the public domain, but Dmitrii Bulanin in a thoughtful review pointed out that a dynastic destination ('for the education of the crown prince,' as I had argued) was unlikely in view of the high incidence of monastic compilations in the period. ${ }^{25} \mathrm{He}$ was right. The evidence of the $\mathrm{D}$ and $\mathrm{E}$ proves me wrong: both compilations had unrestricted access to $\mathrm{C}$, so its limited dissemination is surely no more than accidental. Compilation $\mathrm{E}$, in contrast, shows purpose by assigning texts of $\mathrm{C}$ and $\mathrm{D}$ (and other sources) to Sundays of the ecclesiastical year, obviously as readings complementary to Divine service. E's text versions appear to form the end-point of a protracted process of collectively creating new texts: like other texts of $\mathrm{E}$, interpolation $v$ shows the final touches of their dignification by circumlocution, and interpolation iii shows archaisation by reintroducing the imperative да въси $\rightarrow$ да въждь (inappropriate without deleting да).

That compilations $\mathrm{A} \rightarrow \mathrm{B} \rightarrow \mathrm{C}$ are related in one line of transmission has been noted in earlier studies. That this line can extend to $\mathrm{E}$ as well is proven by the evidence of the exhortation on reading, which shows that $\mathrm{D} \rightarrow \mathrm{C} \rightarrow \mathrm{E}$ must be recognised as another such line of transmission, both lines intersecting in C. The evidence of the exhortation also shows that a line $\mathrm{D} \rightarrow \mathrm{B} \rightarrow \mathrm{E}$ may have existed: in interpolation iii D's text could well be the source of B' ${ }^{26}$ rather than the other way around; in any case, the B $\rightarrow E$ part of that line is attested in E's appropriation of B's chapters 3 and 4 , respectively for the 29 th and 8th Sunday after Trinity.

25 D.M. Bulanin, 'Izbornik 1076 goda i spory o natsional'nykh primetakh v drevneishikh slavianskikh perevodakh,' Russkaia literatura 2 (2012)2: 3-30. His critique, of course, entails that the dates I proposed for A-C, viz. 'ca. 90o', 'ca. 930' and 'ca. 960' must be dismissed.

$26 \quad$ B and D need to be reread carefully over the entire length of their shared text to discover signs of directionality in transmission. 
The development of the compilation technique from an analytic to a synthetic treatment of the source texts together with the (possibly double) intersection of the lines of transmission suggests that the compilers of A-E did not work at great remove from one another.

\section{The Dating of Compilations $A-E$}

A is attested in manuscripts of the twelfth - seventeenth century, B of the fourteenth - sixteenth century, C of the eleventh - seventeenth century, D of the fifteenth - seventeenth century, E of the fourteenth - seventeenth century. Does this mean that $\mathrm{C}$ precedes $\mathrm{A}$, which precedes $\mathrm{B}$ and $\mathrm{E}$ and is succeeded by $\mathrm{D}$ ? No: dating texts by their earliest attestation (not to mention locating them by its origin) is more folklore pageant than scientific endeavour. It is the directionality of transmission that firmly places $\mathrm{A}$ before $\mathrm{B}, \mathrm{B}$ before $\mathrm{C}, \mathrm{B}$ and $\mathrm{C}$ before $\mathrm{E}$, and $\mathrm{D}$ before $\mathrm{C}$ (possibly B, too) and $\mathrm{E}$.

$\mathrm{C}$ is the dated intersection of the lines of transmission: its copy of 1076 reproduces an antigraph more than a century senior, which had used A, B, and D as sources. This entails that A-D must be dated to the tenth century and that only $\mathrm{E}$ is left in limbo.

D was written in Glagolitic script, as testified by the reproduction of the rare

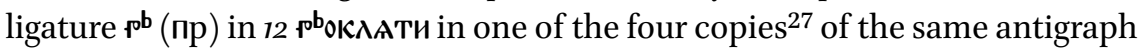
and the misreadings $\sigma_{0} \rightarrow \mathcal{Q}(\mathbf{T} \rightarrow \mathrm{B})$ in $15 \mathrm{BZC} \mathrm{BATb} \rightarrow$ Bocn'BBZ in another, ${ }^{28} \mathrm{ob} \rightarrow$ \& $(\mathrm{A} \rightarrow \boldsymbol{\lambda})$ in iii ПравьА, $\mathbb{R} \rightarrow$ Правилоу in all four. E, too, was written in Glagolitic script as testified by the confusion $\% \leftrightarrow \leftrightarrow(M \leftrightarrow X)$ in 22 свАтZінХZ кZнигаХZ

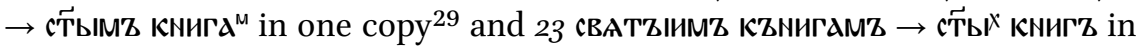
the other ${ }^{30}$ from the same antigraph, the misreadings $r_{0} \rightarrow ?(\Gamma \rightarrow \kappa)$, o $\rightarrow \infty$ o

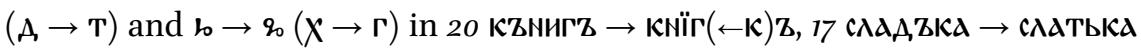

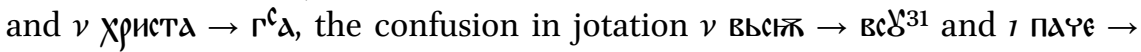

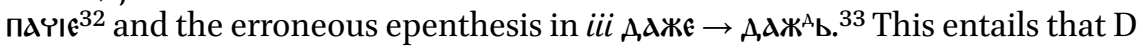
and $E$ cannot be dated later than the early twelfth century. ${ }^{34}$ It also entails that $\mathrm{A}-\mathrm{C}$ as well as all their sources were written in Glagolitic script.

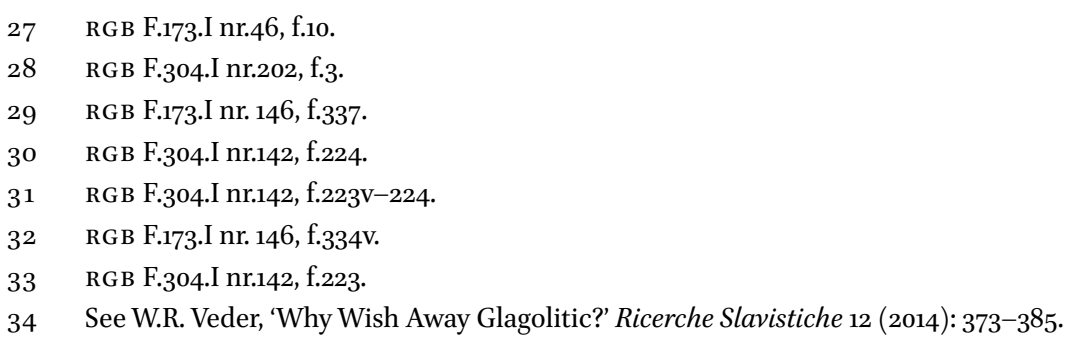




\section{An Efflorescence of Compilations}

The compilations A-E share excerpts with five others: 1 . an excerpt version of the Egyptian Patericon ${ }^{35}$ (a Greek conflation of the Historia monachorum in Aegypto and the De gentibus Indiae et Bragmanibus and Historia Lausiaca by Palladius of Helenopolis); 2. one or two excerpt versions of the Scala Paradisi, like 1. above, used in the compilation of 3 . the Scaliger Patericon ${ }^{36}$ (an excerpt version of the Scete Patericon ${ }^{37}$ ); 4 . the Tikhanov Patericon (an excerpt version of the Scete Patericon with additions from a host of other translations, among which the Alphabetico-Anonymous Patericon ${ }^{38}$ ); and 5. the Zlatostruj (a Slavonic compilation of excerpts from and full texts of translated Chrysostomian homilies $\left.{ }^{39}\right)$. Their compilation technique is shared by another five: 6 . the Limonis (an excerpt version of the Pratum Spirituale by John Moschus), 7. the Svodnyj Paterik (a conflation of excerpts from the Paterika mentioned with addition of edificatory narratives ${ }^{40}$ ); 8 . the Merilo Pravednoe ${ }^{41}$ (which contains an excerpt version of the translated Pchela ${ }^{42}$ ); and 9-10. the Prolog (a compilation from the translated Synaxarium (a) extant in two versions, viz. b, the compilation of a with additional vitae and edifying texts, many of them hailing from the Zlatostruj, and $\mathbf{c}$, the addition to a of edifying texts selected from $\mathbf{b}^{43}$ ). These compilations form a corpus with a length of close to 400,000 words, i.e. more than fivefold that of texts composed in Slavonic in the ninth-tenth centuries, and it is by no means excluded that new identifications of compilations will add to it.

35 Ed. U.R. Feder, 'Paterik Egipetskij Kratkij, peredelka Egipetskogo Paterika do 1076 goda,' in Feder, Hiliada..., 195-210.

36 Ed. R. Nowak and W.R. Veder, The Protopatericon Scaligeri, (Nijmegen 1985) (= Polata knigopisnaja 12), see also W.R. Veder, 'Elementary Compilation in Slavonic', in Feder, Hiliada..., 110-119.

37 Ed. U.R. Feder, Skitskii Paterik, (Amsterdam 2012) (= Pegasus Oost-Europese Studies 14).

38 See U.R. Feder, 'Tikhanov Paterik,' in Feder, Hiliada..., 248-256.

39 See Ya. Miltenov, Zlatostruj, starob"lgarski homiletichen svod, s"zdaden po iniciativa na b"lgarskiia tsar Simeon. Tekstologichesko i izvorovedchesko izsledvane. (Sofia: Avalon, 2013). Study and partial ed. S. Nikolova, Paterichnite razkazi $v$ b"lgarskata srednovekovna literatura. (Sofia: Bulgarskata akademiia na naukite, 1980).

41 Ed. M.N. Tikhomirov, Merilo Pravednoe po rukopisi XIV veka (Moskva: Izd. AN sssR, 1961).

42 Ed. A.A. Pichkhadze et al., Pchela. Drevnerusskii perevod, t. 1-2. (Moskva: Rukopisnye pamiatniki Drevnei Rusi, 2008); see the review W.R. Veder, 'A Retrial for the Pchela,' Polata knigopisnaia 38 (2010):145-154.

43 Ed. L.V. Prokopenko et al., Slaviano-russkii Prolog po drevneishim spiskam: Sinaksar,' Sentiabr'-fevral', t. 1-2. (Moskva: Azbukovnik, 2010-2011); see the review W.R. Veder, 'Markup in the Prolog,' Polata knigopisnaia 39, [forthcoming]. 
The evidence of the transmission of the Scete Patericon indicates that a first copy ( + ) of the protograph was made not long after it was brought to Pliska in 886 and left unbound for ease of copying: five copies of it are attested $(\alpha-\delta)$, progressively muddling the order of the quires: ${ }^{44}$ the Scaliger Patericon follows $\alpha$, the Tikhanov Patericon follows $\delta$, and both attest development, the first leaving selection entirely to chance, the second showing rudimentary discrimination. It is highly probable that both compilations were made by the men who had written the fair copies of $\alpha$ and $\delta$, and it is just as probable that they were made even before the ninth century was over. The evidence of the transmission of the Zlatostruj indicates that its original, too, may have been left unbound for ease of copying; the transmission of the excerpts, in addition, shows that they may have existed as such separately from the homilies. ${ }^{45}$

The evidence of the transmission of the sixteen compilations referred to above shows that they were all written in Glagolitic script (as were their sources), which precludes any of them postdating the beginning of the twelfth century.

As to the environment in which these compilations were made, I think we can trust the colophon of John the Sinner (at the end of compilation C), who says that he compiled his book 'from the many books of the prince'. This points to a physical crossroads, which may well be the library of Prince Boris-Michael at Pliska and, after 893, that of Prince (later tsar) Simeon at Preslav. After 971, we must think of the library at Ohrid: the Greek Synaxarium, compiled after 992, was translated when the Preslav library had been removed to Kiev; version $\mathbf{b}$ of the Prolog reflects a variety of sources more limited than that of its predecessors.

Both the contacts between the individual compilations and the development of the compilation techniques suggest that the compilers must have been in contact with one another and mutually fertilised their endeavours. Compilation as an art form reaches a high point before 971 in the Zlatostruj and the homilies of John the Exarch, which freely compile selections purposely translated from Greek homilies. ${ }^{46}$

44 See W.R. Veder, The Scete Patericon, Amsterdam 2012 (= Pegasus Oost-Europese Studies 12), 34-35.

45 See Ya. Miltenov, 'Obshtite pasazhi mezhdu kolekciiata Zlatostruj i Kniazheskiia Izbornik,' Starob"lgarska literatura 49-50 (2014): 28-45.

46 For John the Exarch, see M. Capaldo, 'Jean l'Exarque en tant que compilateur et traducteur,' Polata knigopisnaia 3 (1980): 69-89; id. 'Art compilatoire et technique de traduction dans l'homélie de Jean le Prêtre sur le Baptême du Seigneur,' Polata knigopisnaia 8 (1983): 2-14; id. 'La source principale du Sermon sur la Nativité attribué à Jean l'Exarque,' Polata knigopisnaia 9 (1984): 3-29. 


\section{In Lieu of a Conclusion}

The compilations reviewed above are unquestionably no 'poor relations' to 'real' literature. They are so diverse and voluminous that it will take the efforts of many researchers with different interests to sort out their origins, interrelations and transformations, tasks which require great attention to detail. Such work will be as rewarding as the study of full translations and original compositions, if only because the transformations of the texts in the hands of successive compilers provide invaluable data as to the contemporary reception of their content and means of expression. At present, only the most pressing questions of general import can be asked: (1) Why do compilations assume a space which could be occupied by original Slavonic compositions? (2) Does their massive appearance in the ninth-eleventh century bear testimony only to active acquisition and digestion of texts, or does it have a bearing on their language as well? (3) Why are these compilations not discarded over time but transmitted well into the sixteenth-seventeenth centuries? 